\title{
Review on Multi-Scale Models of Solid-Electrolyte Interphase Formation
}

\author{
Birger Horstmann ${ }^{\mathrm{a}, \mathrm{b}}$, Fabian Single $\mathrm{a}^{\mathrm{a}, \mathrm{b}}$, Arnulf Latz $\mathrm{z}^{\mathrm{a}, \mathrm{b}, \mathrm{c}}$ \\ ${ }^{a}$ Helmholtz Institute Ulm (HIU), Helmholtzstraße 11, 89081 Ulm, Germany \\ ${ }^{b}$ German Aerospace Center (DLR), Institute of Engineering Thermodynamics, Pfaffenwaldring 38-40, 70569 Stuttgart, Germany \\ ${ }^{c}$ Ulm University, Institute of Electrochemistry, Albert-Einstein-Allee 47, 89069 Ulm, Germany
}

\begin{abstract}
Electrolyte reduction products form the solid-electrolyte interphase (SEI) on negative electrodes of lithium-ion batteries. Even though this process practically stabilizes the electrode-electrolyte interface, it results in continued capacity-fade limiting lifetime and safety of lithium-ion batteries. Recent atomistic and continuum theories give new insights into the growth of structures and the transport of ions in the SEI. The diffusion of neutral radicals has emerged as a prominent candidate for the long-term growth mechanism, because it predicts the observed potential dependence of SEI growth.
\end{abstract}

\section{Highlights}

- Solid-electrolyte interphase passivates negative electrodes in lithium-ion batteries

- Recent models elucidate dynamics of solid-electrolyte interphase

- Multiple theoretical methods employed: from quantum theory to thermodynamics

- Continued capacity fade due to diffusion of neutral radicals

Keywords: lithium-ion battery, solid-electrolyte interphase, SEI growth, capacity fade, multi-scale modeling, validation

\section{Introduction}

Standard lithium-ion batteries rely on graphite as negative electrode material even though graphite decomposes the standard electrolytes at their working potentials (see figure 1). The decomposition products form the so-called solid-electrolyte interphase (SEI) which is protecting the electrolyte and suppresses further electrolyte reduction [4, 5]. Nevertheless, lithium transport through the SEI remains possible and is typically not limiting battery performance. The SEI is in the focus of many processes limiting lifetime, performance, and safety of lithium-ion batteries. It affects the inhomogeneous growth and dissolution of lithium metal $[6,7]$. Thermal runaway as the main cause for battery failure is promoted by SEI decomposition $[8,9,10,11]$. The main capacity fade during battery storage stems from the consumption of lithium due to the continued growth of SEI [12, 13]. During battery cycling, graphite undergoes a notable volume change damaging the SEI and accelerating loss of cycle-able lithium. This volume change is even more pronounced for next-generation high-capacity materials like lithium metal or silicon [14]. Generally, the quest

Email address: birger.horstmann@dlr.de (Birger Horstmann) for larger battery cell voltages requires improvements in interfacial stability. Thus, SEI modeling contributes to the broad theoretical effort towards rational design of stable electrolytes $[15,16,17]$.

Since 1979 a multitude of experimental SEI research has been performed $[4,18]$, recent examples include battery storage at various state-of-charge (SoC) $[12,13]$, differential capacity analysis during cycling [19], neutron reflectometry [20], atomic force microscopy [21], nuclear magnetic resonance [22], redox shuttles [23, 24], fourier-transform infrared spectroscopy [25], and photo-electron spectroscopy [26]. As a consequence, there is a general understanding of SEI composition and morphology for few specific systems. The chemical composition of the SEI, however, is diverse and disturbed by trace-amounts of contaminants. Therefore, elucidating SEI behavior requires a careful experimental effort and several key questions about basic SEI mechanisms have yet to be answered (see figure 2). Most striking is the fact that the mechanism for $\mathrm{Li}^{+}$transport through the SEI is still debated. A dual-layer structure of SEI is typically described with an inner compact layer and a porous polymeric outer layer [27], but both the thickness and the formation mechanism of these layers are still debated. 


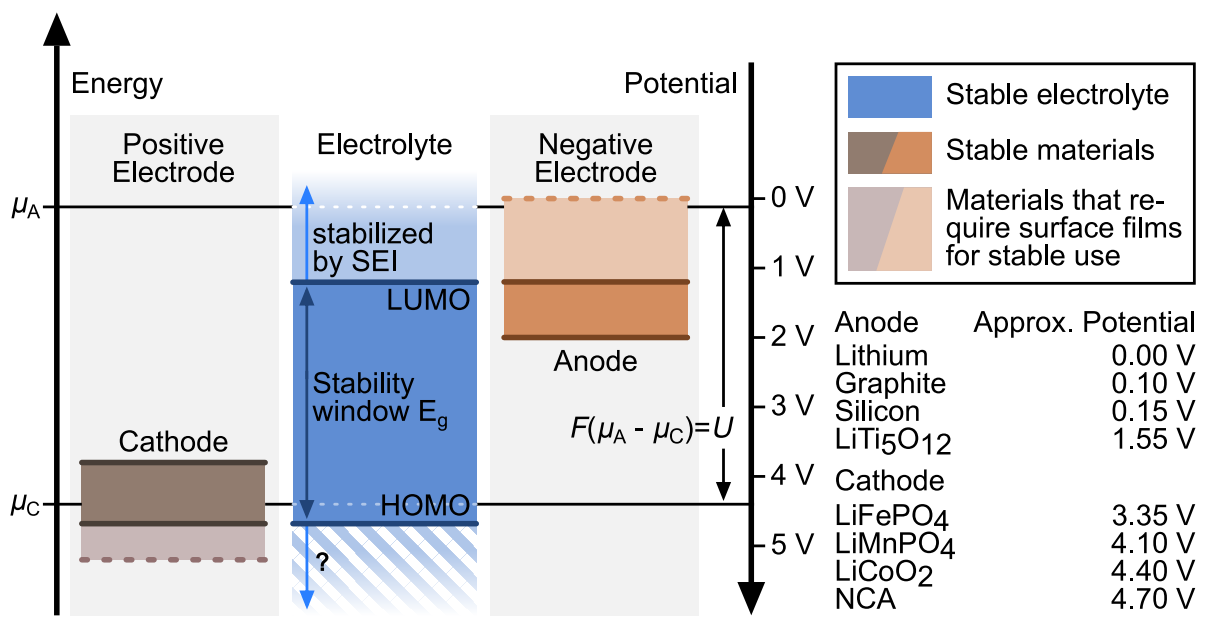

Figure 1. Stability of electrode-electrolyte interface in lithium batteries [1]. The positive electrode (left) operates at low energies or high potentials (measured in galvanostatic measurements in EC-PC/LiPF6 solutions at C/20 rates [2]), whereas the negative one operates at high energies/low potentials [3]. The stability window is extended by surface films, denoted solid-electrolyte interphase (SEI) on negative electrodes. Stable interfaces are the key for the realization of next-generation low-voltage negative electrodes and high-voltage positive electrodes.

Under these circumstances, theoretical studies provide important complementary insights into universal principles of SEI chemistry, structure, and dynamics. The diversity of entangled length and time scales governing SEI properties constitutes a fundamental theoretical challenge. One should, for example, distinguish between the process of initial SEI formation in hours and days and the continued SEI growth in months and years. On the one hand, SEI chemistry is governed by reactions between individual atoms and molecules. On the other hand, molecular environments influence reaction pathways and transport through the SEI determines the availability of reactants. Therefore, we begin with a brief summary of results from atomistic theories based on quantum physics as they are prerequisites for multi-scale models on larger scales.

This review, however, focuses on recent continuum models based on thermodynamics. These meso-scale models discuss emergent phenomena of SEI formation, particularly, the origin of continued SEI growth. SEI thickness is experimentally observed to grow with the square-root of time during storage under controlled lab conditions. Therefore, a transport process seems to limit SEI growth after sufficiently long times. Continuum models evaluate various long-term growth mechanisms

a) Electron tunneling [28, 29]

b) Diffusion of solvent/salt molecules/anions [30, 14, 31, 32, $28,33,34,35,36,37,38]$

c) Electron conduction or diffusion $[39,40,41,28,34,35$, 42, 38, 43]

d) Diffusion of neutral radicals such as lithium interstitials $[44,45,35,38,43]$

Most models describe the ideal square-root-of-time dependence of capacity fade. Electron tunneling, however, predicts capacity fade with the logarithm of time as discussed below. Some articles model battery operation and analyze linear growth regimes. In this review, we highlight models that predict additional ob- servable properties, i.e., morphology of SEI [28, 34, 35], explain additional dependencies, i.e., potential dependence of SEI growth [38, 43], or analyze non-ideal settings, i.e., SEI growth during cycling [43]. These allow the experimental validation of proposed growth mechanisms.

\section{Atomistic theories and initial SEI growth}

Atomistic simulation methods address elementary reaction and transport processes in the SEI. Energies of atom configurations in electrolytes are probed with quantum chemistry and density functional theory (DFT). The resulting energy landscape determines forces between atoms and reaction probabilities. The collective dynamics of molecules and atoms can then be calculated with molecular dynamics simulations (MD). In this section, we give a brief outline of results from atomistic simulations, but refer to recent reviews for further details [46, 47, 48, 49].

Borodin et al. highlight general challenges for calculations of electrolyte stability [50]. Solvent and solutes interact so strongly that calculations on individual molecules are inaccurate. This necessitates large simulation domains and optimized molecular geometries. The diverse SEI chemistry imposes further challenges. It has been shown with DFT and ab-initio MD that salt anion [51, 25] and electrode voltage [52] affect electrolyte stability and chemical SEI composition. Nevertheless, recent calculations provide further insights into preferred reduction pathways in conventional lithium battery electrolytes comprising a mixture of ethylene carbonate (EC) and linear carbonates, e.g. dimethyl carbonate (DMC). In agreement with experimental observations, it is rationalized that EC is preferentially reduced because $\mathrm{EC}$ has a higher reduction potential than DMC [50], EC preferential adsorbs on the SEI surface [53], and $\mathrm{Li}^{+}$prefers EC in its inner solvation shell [54].

Atomistic theories alone can only address the initial stages of SEI formation because of limits in simulated space and 
a)

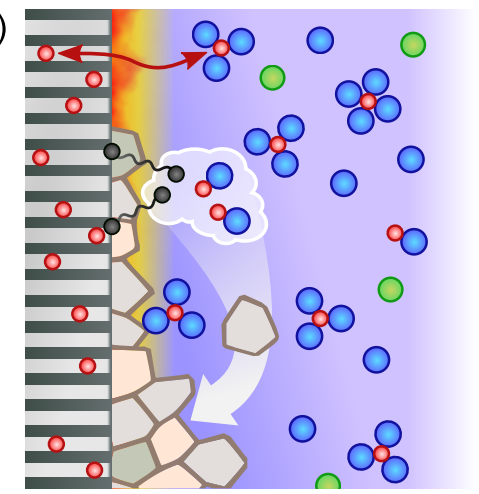

b)

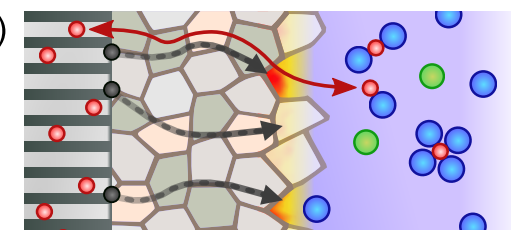

c)

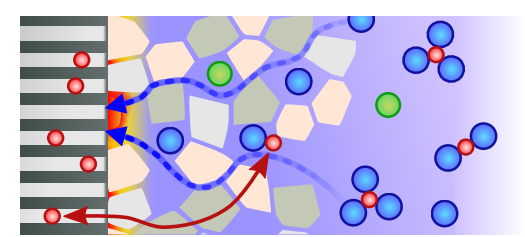

$\mathrm{e}^{-}$

$\circ \mathrm{Li}^{+}$

$\mathrm{A} \mathrm{A}^{-}$

OEC

Electrolyte

$\square$ SEI

Graphite

$\checkmark$ SEl formation Reaction

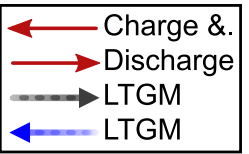

Figure 2. Cross-section through the negative electrode, the SEI, and the electrolyte. Solvent, Li ions and electrons are mobile species and move as indicated by the corresponding arrows. (a) Initial SEI formation: Electrons tunnel, electrolyte is reduced and reduction products precipitate as solid film. (b) Long-term SEI growth proceeds via a mechanism that transports negative charge to the SEI/electrolyte interface. (c) Alternatively, long-term SEI growth is caused by electrolyte diffusing towards the electrode/SEI interface.

time $[55,56]$. Electron tunneling allows the transport of electrons through 2-3 nm thin SEI layers [57], while SEI thickness quickly exceeds $10 \mathrm{~nm}[58,27]$. This suggests that electron tunneling plays a role only in the initial part of first-cycle SEI growth (see fig. 2a). $\mathrm{Li}_{2} \mathrm{O}$ is predicted to form the innermost SEI layer on the electrode surface at low potentials [59]. Furthermore, nucleation and precipitation play an important role in the initial SEI formation [60].

Furthermore, the mechanism for $\mathrm{Li}^{+}$transport through the SEI is analyzed with atomistic calculations. For the inner inorganic layer, different lattice diffusion mechanisms in crystalline $\mathrm{LiF}, \mathrm{Li}_{2} \mathrm{O}$ and $\mathrm{Li}_{2} \mathrm{CO}_{3}$ are compared [44, 61, 62, 63]. Alternatively, $\mathrm{Li}^{+}$is proposed to diffuse along interfaces between these crystalline phases [64]. For the outer organic layer, MD determines diffusion constants of $\mathrm{Li}^{+}$through ordered and disordered LiEDC [65]. Besides transport of $\mathrm{Li}^{+}$, atomistic theories discuss mechanisms for electron transport in the SEI. We highlight the recent proposals of diffusion of neutral lithium interstitials through the crystalline inner layer [44, 61] and radical diffusion through the polymeric and amorphous outer layer [45]. These mechanisms lay the foundation for novel models of continued SEI growth (see Sec. 4).

\section{Continuum models and long-term SEI growth}

In 2001, Broussely et al. recorded the lifetime of lithiumion batteries and observed a continued capacity fade due to SEI growth [39]. Assuming transport-limited SEI growth and neglecting the electrochemical details, they derive a rate equation for SEI thickness evolution. This prototype model demonstrates that sluggish electron transport through the SEI would explain the observed square-root-of-time behavior of capacity fade. Subsequent modeling studies elaborate on this model and present various long-term growth mechanisms (LTGM) $[40,30]$. On the one hand, the coupled diffusion and/or migration of negative charges, e.g. electron conduction, from the graphite/SEI interface to the SEI/electrolyte interface predicts the observed SEI growth [40]. (see fig. 2b). On the other hand, the diffusion of electrolyte constituents, e.g. solvent molecules, from the SEI/electrolyte to the graphite/SEI interface agrees equally well with SEI thickness evolution [30] (see fig. 2c). Note that the core mathematical description of SEI thickness is equivalent for both LTGMs. To conclude, continuum models should predict measurable properties beyond SEI thickness in order to determine the LTGM.

A coupled multi-species model found a minor influence of cycling on SEI thickness [41]. Cell-level models conclude that SEI thickness varies little in a porous electrode $[14,36]$. Pinson and Bazant extend their SEI model and describe the rapid capacity decrease during cycling [14]. Because the drastic volume change of silicon electrodes stresses the SEI, a constant rate of SEI cracking is assumed. Therefore, SEI thickness deviates from the square-root-of-time law and grows linearly in time, as observed experimentally on silicon anodes. Coupled models of continuum mechanics and electrochemistry begin to take a closer look at SEI fracture [66]. The combination of continuum simulations of transport with stochastic Monte Carlo simulations of reduction reactions gives further microscopic insights, but has not yet lead to new macroscopic predictions [33, 37, 42].

Some articles analyze the role of electron tunneling for longterm SEI growth. Because capacity fade would grow with the logarithm of SEI thickness, Tang et al. discard electron tunneling as possible LTGM [28]. Nevertheless, a model based on electron tunneling has recently been fitted to capacity fade experiments [29]. In this model, the growth of the outer SEI layer is controlled by electron tunneling through an approximately $3 \mathrm{~nm}$ thin inner layer. The ratio of growth of the inner versus the outer layer is determined by model assumption such that the inner layer does not even grow a single mono-layer during the long-term experiment. We note that the capacity fade experiments discussed in Ref. [29] can be fitted equally well with square-root-of-time-growth as with logarithm-of-time-growth. This demonstrates that time dependence of capacity fade as single metric cannot prove the correctness of a SEI growth model.

In a comprehensive experimental and theoretical approach, Tang et al. study SEI formation and redox shuttles at negative electrodes $[31,32,28]$. A dual-layer SEI with a compact inner and a porous outer layer is modeled with a volume- 
averaged transport theory by introducing a constant porosity in each layer $\varepsilon$. They aim at determining the LTGM by comparing experiments with different models, each based on a single rate-limiting mechanism [28]. The square-root-of-time behavior restricts possible LTGMs to transport mechanisms, e.g., solvent diffusion and electron conduction. Solvent diffusion fails to explain the observed dependence of growth rate on electrode potential. Electron conduction fails to explain the involvement of convection in SEI growth. They finally conclude that another form of charge transport must be rate-limiting.

The recent models of Single et al. take into account two counter-propagating transport processes, i.e., motion of charges from the electrode to the electrolyte and motion of solvent molecules from the electrolyte to the electrode [34, 35]. This allows to predict not only SEI thickness, but also SEI porosity $\varepsilon(x, t)$. A volume-averaged transport model determines the spatially-resolved dynamics of solvent, electric potential, and SEI porosity. Modeling convection of solid SEI facilitates simulating reduction reactions inside the SEI.

A single-layer SEI comes out if solvent EC is reduced to $\mathrm{Li}_{2} \mathrm{EDC}$ and co-solvent DMC is inert [34, 35]. A typical evolution of SEI volume fraction is depicted in fig. 3a1. It is found that SEI growth is limited by electron transport and that SEI predominantly grows at the electrolyte/SEI interface [34] ( $\tilde{\Pi} \approx 1$ in fig. 3c). Therefore, SEI thickness grows like the square-root-of-time in agreement with capacity fade experiments (see fig. 3b). The predicted SEI porosity is almost constant and approaches a stability point $\varepsilon^{*}$ determined by electrolyte transport properties. The transition from electron conduction to solvent diffusion as LTGM is studied by imposing large SEI porosity and taking into account solid convection [35]. If solvent diffusion is rate-limiting, the reaction zone moves to the electrode/SEI interface ( $\tilde{\Pi} \approx 0$ in fig. $3 \mathrm{c}$ ) and significant fluctuations in SEI thickness are predicted.

Additional SEI formation reactions lead to a dual-layer SEI [35]. If reduction of co-solvent DMC or primary SEI compound $\mathrm{Li}_{2} \mathrm{EDC}$ is considered, low potentials favor the second reduction near the electrode and a compact, non-porous, inner layer is formed (see fig. 3a2,a3). The ratio between the thickness of inner and outer layer is determined by electrode potential and material parameters. Simulations illustrate that this stationary thickness ratio is quickly re-attained after the SEI is disturbed. Most importantly, SEI thickness and capacity fade grow with the square-root of time for dual-layer morphologies, as well.

\section{Multi-scale models of electron leakage via neutral radi- cals}

Based on atomistic theories, Shi et al. and Soto et al. propose diffusion of neutral radicals as an alternative mechanism for charge transport from the electrode through the SEI into the electrolyte [44, 45]. In the case of inorganic SEI, lithium ions take up an electron at the electrode/SEI interface, diffuse as neutral lithium interstitials through the SEI, and release an electron at the SEI/electrolyte interface [44]. In the porous organic SEI, radicals formed by electrolyte reduction can act as electron carrier [45]. Single et al. take up this result and develop a con- a)

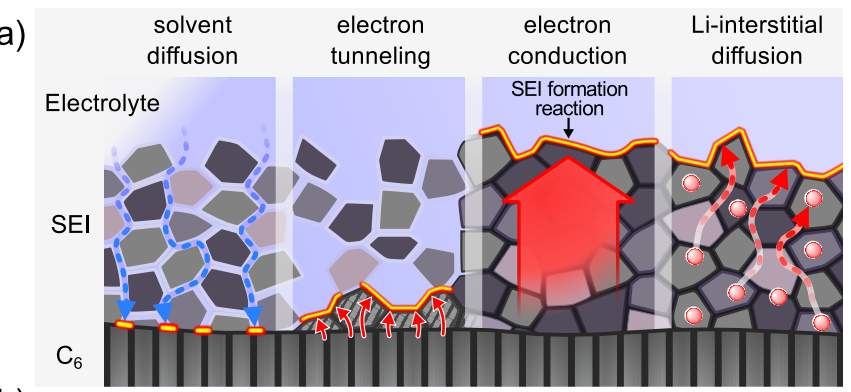

b)
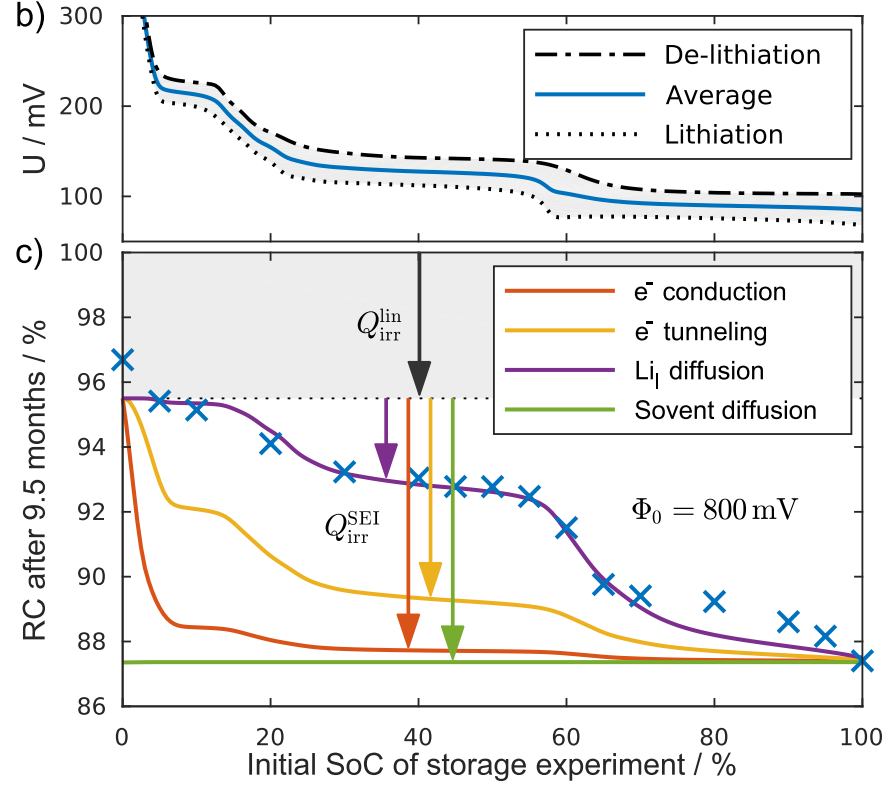

Figure 4. (a) Schematic of all four candidates for transport mechanisms: Solvent diffusion through small SEI pores, electron tunneling through a thin and dense inner SEI layer, electron conduction through the SEI, diffusion of neutral Li-interstitials through the SEI. The SEI formation reaction takes place at different interfaces depending on the mechanism, marked yellow/red. (b) Open circuit voltage of the negative electrode gained by averaging the lithiation and delithiation voltages (half cell, cycled at C/20). (c) Experimentally obtained relative capacity after 9.5 months of storage (crosses) compared to that predicted by four different long-term growth mechanisms (lines). Reproduced from Single et al. [38].

tinuum model based on diffusion of neutral radicals [35]. SEI profiles simulated with this mechanism share the same features as those described above for electron conduction. Recent continuum models highlight the unique exponential dependence of SEI growth rate on electrode potential for this mechanism $[38,43]$.

The first such model by Single et al. points out that the concentration of radicals at the electrode is determined by its electric potential [38]. They compare the predictions of different LTGMs with capacity fade experiments for various graphite potentials and state-of-charges (SoC) [13]. Simple theories based on the four LTGMs enlisted above are created: electrolyte diffusion, electron tunneling, electron conduction, and lithium interstitial diffusion. As summarized in fig. 4, solvent diffusion does not reproduce any SoC dependence. The SoC dependence of electron conduction and electron tunneling does not agree with the experiment for any reasonable choice of parameters. Only a mechanism such as neutral lithium interstitial diffusion 

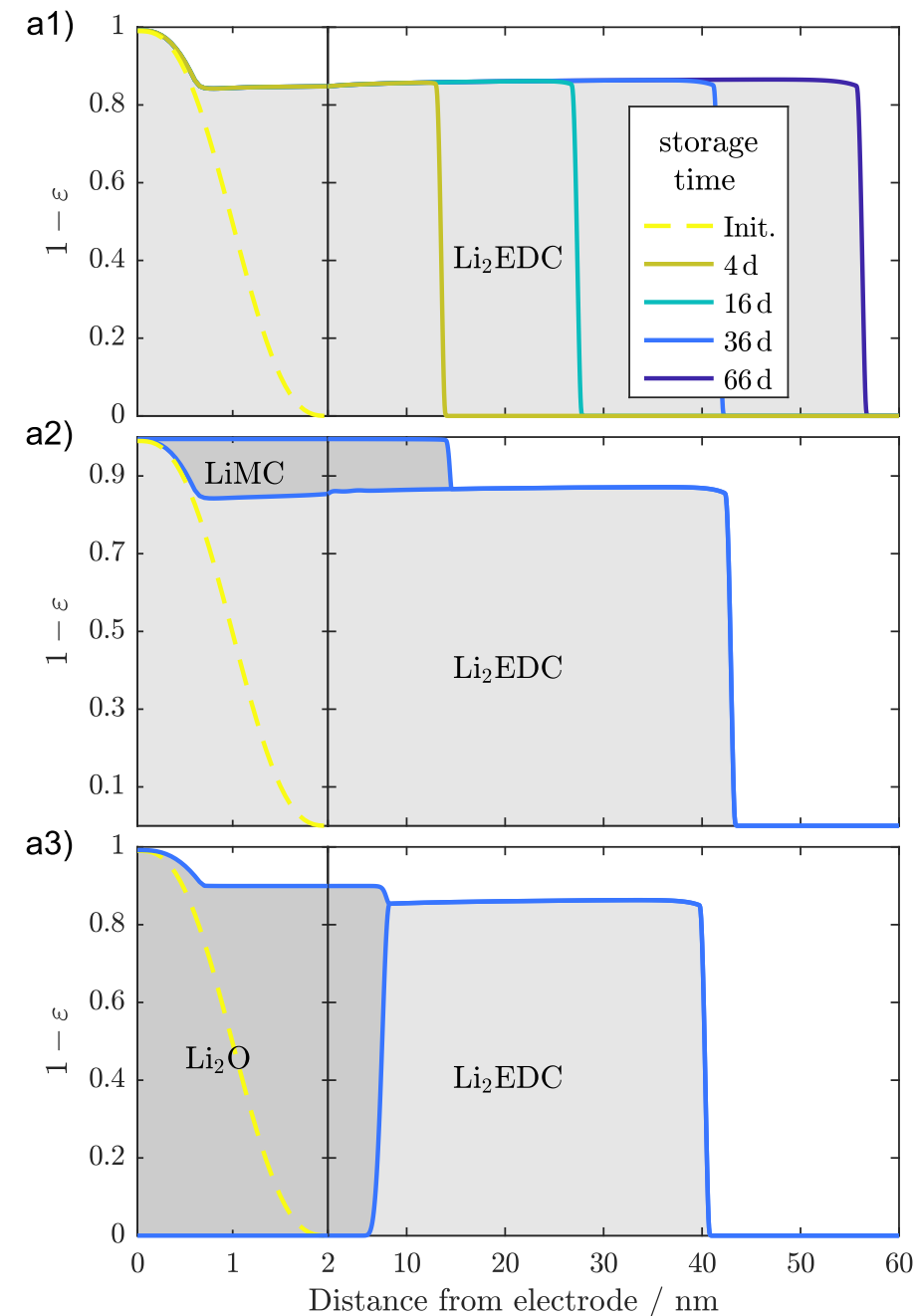
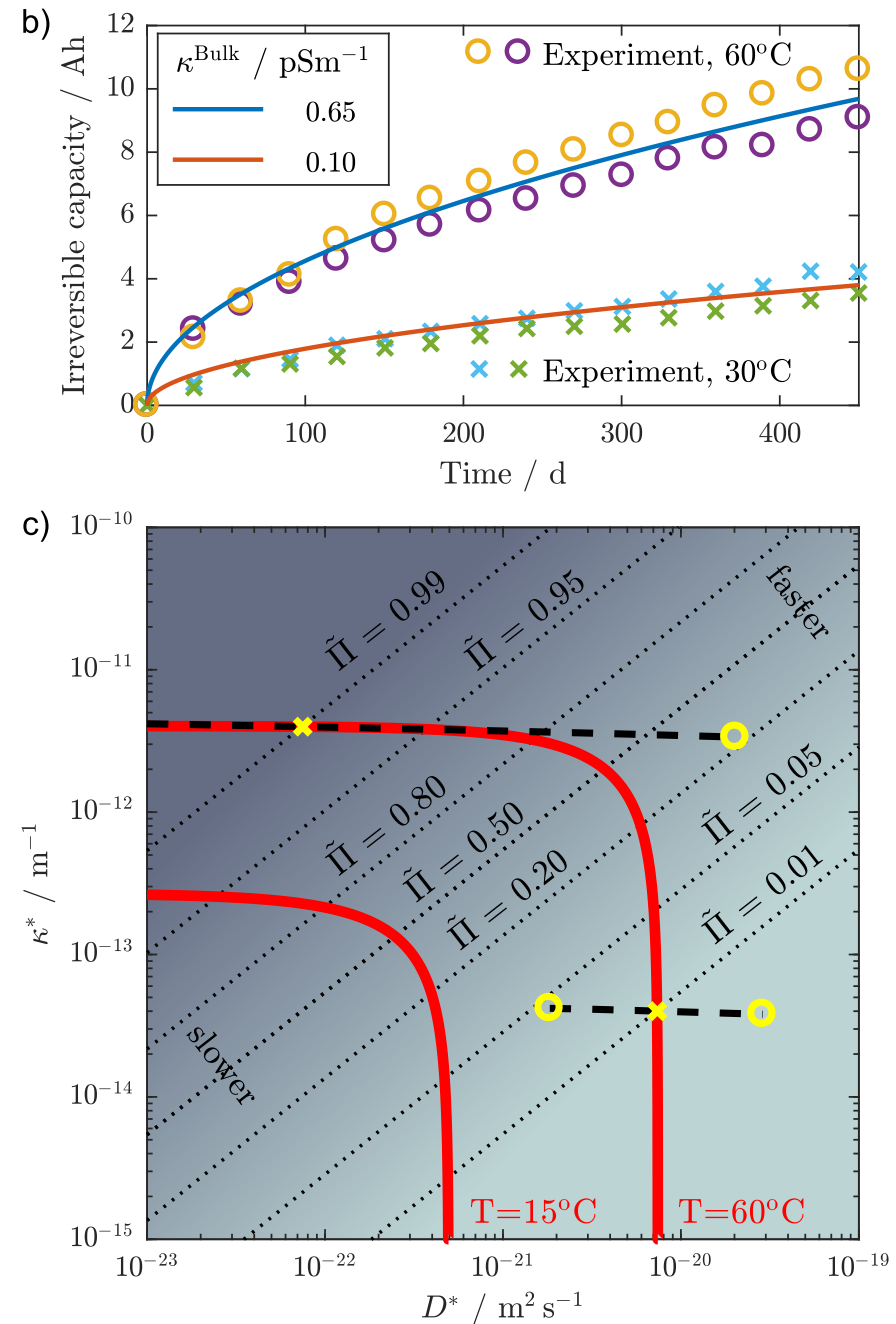

Figure 3. Simulation of long-term SEI growth. (a) Time evolution of the SEI volume fraction for (a1) a single-layer SEI from reduction of EC to Li 2 EDC, (a2) a dual-layer SEI due to reduction of co-solvent DMC, and (a3) a dual-layer SEI due to conversion of $\mathrm{Li}_{2} \mathrm{EDC}_{\text {. }}$ (b) Simulated capacity fade according to the electron conduction mechanism with conductivity $\kappa^{\text {Bulk }}$ (lines) compared to experimental data (circles and crosses) [39] close to the upper yellow cross in (c). (c) Relative position of the reaction interface $\tilde{\Pi}$ depending on the effective transport parameters $D^{*}$ and $\kappa^{*}$. The red lines show parameter sets with identical SEI growth rates. The dashed black lines end in yellow circles where the formation rate is double (right) or half (left) of the original growth rate. Reproduced from Single et al. [35].

results in a promising agreement with the experiment and remains a candidate for the LTGM.

Recently, Das et al. extend this model and couple SEI growth with lithium-ion transport through the SEI [43]. Based on atomistic theories [44], they assume diffusion of lithium ions on interstitial sites and electron conduction on this sparse network of lithium-ion interstitials. Note that an electron bound to a lithium-ion interstitial constitutes the aforementioned neutral lithium interstitial. As a consequence, the concentration of lithium ions determines electron conductivity. This model can explain recent differential capacity measurements that SEI grows only during lithiation, but not during delithiation [19].

\section{Conclusions}

In this short review, we summarize recent theoretical studies of SEI structure and formation. A multi-scale approach is necessary to elucidate the broad range of SEI properties from chemical composition to mechanical structure. Predictions of atomistic theories converge towards a clear SEI chemistry for standard carbonate-based electrolytes, but the relevance of transport mechanisms remains debated.

Continuum models build on recent findings and demonstrate macroscopically observable consequences of microscopic material behavior. Understanding SEI formation is a key goal. We distinguish between formation of initial SEI and long-term SEI growth. Recent simulations explain the SEI dual-layer structure. Diffusion of neutral radicals leads to the observed potential dependence of long-term growth mechanisms. Coupling this mechanism with lithium-ion diffusion predicts an observed asymmetry in SEI growth during cycling.

\section{Acknowledgement}

This work is supported by the German Federal Ministry of Education and Research (BMBF) in the project Li-EcoSafe 
(03X4636A). Further support was provided by the bwHPC initiative and the bwHPCC5 project through associated compute services of the JUSTUS HPC facility at the University of Ulm. This work contributes to the research performed at CELEST (Center for Electrochemical Energy Storage Ulm-Karlsruhe).

\section{Highlighted References}

[28] ** M. Tang, S. Lu, J. Newman, J. Electrochem. Soc. 2012, 159, A1775-A1785. ** In a comprehensive theoretical and experimental approach, Tang et al. compare various transport mechanisms as possible long-term growth mechanism.

[35] * F. Single, B. Horstmann, A. Latz, J. Electrochem. Soc. 2017, 164, E3132-E3145. * This paper models not only SEI thickness, but also SEI porosity and SEI dual-layer structure. The transition from electron conduction to solvent diffusion as long-term growth mechanism is studied.

[38] ** F. Single, A. Latz, B. Horstmann, ChemSusChem 2018, 11, 1950-1955. ** In this article, Single et al. compare the SoC dependence of various long-term growth mechanisms with storage experiments and present the first indirect experimental evidence for neutral interstitial diffusion.

[43] * S. Das, P. M. Attia, W. C. Chueh, M. Z. Bazant, arXiv 2018, to be submitted. * By coupling neutral lithium interstitials with lithiumion interstitials, the authors explain recent experiments showing asymmetric SEI growth during (de-)intercalation.

[44] ** S. Shi, P. Lu, Z. Liu, Y. Qi, L. G. Hector, H. Li, S. J. Harris, J. Am. Chem. Soc. 2012, 134, 15476-15487. ** This article presents atomistic calculations of transport mechanisms in inorganic SEI material. For the first time, it proposes diffusion of neutral lithium interstitials as SEI growth mechanism.

[45] * F. A. Soto, Y. Ma, J. M. Martinez De La Hoz, J. M. Seminario, P. B. Balbuena, Chem. Mater. 2015, 27, 7990-8000. * This article proposes the formation of organic radicals which allow electron leakage through the outer organic SEI layer.

[50] * O. Borodin, M. Olguin, C. E. Spear, K. W. Leiter, J. Knap, Nanotechnology 2015, 26, 354003. * Borodin et al. highlight general challenges for atomistic calculations of electrolyte stability.

\section{References}

[1] J. B. Goodenough, Y. Kim, Challenges for Rechargeable Li Batteries, Chemistry of Materials 22 (3) (2010) 587. doi:10.1021/cm901452z.

[2] S. K. Martha, O. Haik, E. Zinigrad, I. Exnar, T. Drezen, J. H. Miners, D. Aurbach, On the Thermal Stability of Olivine Cathode Materials for Lithium-Ion Batteries, Journal of The Electrochemical Society 158 (10) (2011) A1115. doi:10.1149/1.3622849.

[3] T. Ohzuku, R. J. Brodd, An overview of positive-electrode materials for advanced lithium-ion batteries, Journal of Power Sources 174 (2) (2007) 449-456. doi:10.1016/j.jpowsour.2007.06.154.

[4] E. Peled, The Electrochemical Behavior of Alkali and Alkaline Earth Metals in Nonaqueous Battery Systems-The Solid Electrolyte Interphase Model, Journal of The Electrochemical Society 126 (12) (1979) $2047-$ 2051. doi:10.1149/1.2128859.

[5] D. Aurbach, Review of selected electrode-solution interactions which determine the performance of $\mathrm{Li}$ and $\mathrm{Li}$ ion batteries, Journal of Power Sources 89 (2) (2000) 206-218. doi:10.1016/S0378-7753(00) 00431-6

[6] B. Horstmann, F. Single, S. Hein, T. Schmitt, A. Latz, Modeling Graphite Surfaces: Lithium Plating \& Solid Electrolyte Interphase, in: E2Flight Symposium, 2016, pp. 7--8.
[7] A. Kushima, K. P. So, C. Su, P. Bai, N. Kuriyama, T. Maebashi, Y. Fujiwara, M. Z. Bazant, J. Li, Liquid cell transmission electron microscopy observation of lithium metal growth and dissolution: Root growth, dead lithium and lithium flotsams, Nano Energy 32 (2017) 271-279. doi: 10.1016/j. nanoen. 2016.12.001.

[8] H. Maleki, G. Deng, A. Anani, J. Howard, Thermal Stability Studies of Li-Ion Cells and Components, Journal of The Electrochemical Society 146 (9) (1999) 3224. doi:10.1149/1.1392458.

[9] D. P. Finegan, M. Scheel, J. B. Robinson, B. Tjaden, I. Hunt, T. J. Mason, J. Millichamp, M. Di Michiel, G. J. Offer, G. Hinds, D. J. Brett, P. R. Shearing, In-operando high-speed tomography of lithium-ion batteries during thermal runaway, Nature Communications 6 (2015) 1-10. doi $: 10.1038 /$ ncomms7924.

[10] S. Abada, G. Marlair, A. Lecocq, M. Petit, V. Sauvant-Moynot, F. Huet, Safety focused modeling of lithium-ion batteries: A review, Journal of Power Sources 306 (2016) 178-192. doi:10.1016/j. jpowsour. 2015.11.100.

[11] N. Tanaka, W. G. Bessler, Numerical investigation of kinetic mechanism for runaway thermo-electrochemistry in lithium-ion cells, Solid State Ionics 262 (2014) 70-73. doi:10.1016/j.ssi.2013.10.0092013.

[12] P. Keil, S. F. Schuster, J. Wilhelm, J. Travi, A. Hauser, R. C. Karl, A. Jossen, Calendar Aging of Lithium-Ion Batteries, Journal of The Electrochemical Society 163 (9) (2016) A1872-A1880. doi:10.1149/2. 0411609jes.

[13] P. Keil, A. Jossen, Calendar Aging of NCA Lithium-Ion Batteries Investigated by Differential Voltage Analysis and Coulomb Tracking, Journal of The Electrochemical Society 164 (1) (2017) A6066-A6074. doi: 10.1149/2.0091701jes.

[14] M. B. Pinson, M. Z. Bazant, Theory of SEI Formation in Rechargeable Batteries: Capacity Fade, Accelerated Aging and Lifetime Prediction, Journal of the Electrochemical Society 160 (2) (2012) A243-A250. doi: $10.1149 / 2.044302$ jes.

[15] J. Vatamanu, O. Borodin, Ramifications of Water-in-Salt Interfacial Structure at Charged Electrodes for Electrolyte Electrochemical Stability, Journal of Physical Chemistry Letters 8 (18) (2017) 4362-4367. doi: 10.1021/acs.jpclett.7b01879.

[16] V. Hoffmann, G. Pulletikurthi, T. Carstens, A. Lahiri, A. Borodin, M. Schammer, B. Horstmann, A. Latz, F. Endres, Influence of a Silver Salt on the Nanostructure of an $\mathrm{Au}(111)$ Ionic Liquid Interface: an Atomic Force Microscopy Study and Theoretical Concepts, Physical Chemistry Chemical Physics 20 (7) (2018) 4760-4771. doi:10.1039/ C7CP08243F.

[17] M. McEldrew, Z. A. H. Goodwin, A. A. Kornyshev, M. Z. Bazant, Theory of the Double Layer in Water-in-Salt Electrolytes, The Journal of Physical Chemistry Letters 9 (2018) 5840-5846. arXiv:1808.06118, doi:10. $1021 /$ acs. jpclett. 8 b02543.

[18] M. Gauthier, T. J. Carney, A. Grimaud, L. Giordano, N. Pour, H. H. Chang, D. P. Fenning, S. F. Lux, O. Paschos, C. Bauer, F. Maglia, S. Lupart, P. Lamp, Y. Shao-Horn, Electrode-Electrolyte Interface in Li-Ion Batteries: Current Understanding and New Insights, Journal of Physical Chemistry Letters 6 (22) (2015) 4653-4672. doi:10.1021/acs. jpclett.5b01727.

[19] P. M. Attia, S. Das, K. Lim, M. Z. Bazant, W. C. Chueh, Electrochemical kinetics of SEI growth in Li-ion batteries Part I: Experimental quantification of voltage, current rate, and current direction dependence on carbon anodes, private communication.

[20] M. Steinhauer, M. Stich, M. Kurniawan, B.-K. Seidlhofer, M. Trapp, A. Bund, N. Wagner, K. A. Friedrich, In Situ Studies of Solid Electrolyte Interphase (SEI) Formation on Crystalline Carbon Surfaces by Neutron Reflectometry and Atomic Force Microscopy, ACS Applied Materials \& Interfaces 9 (41) (2017) 35794-35801. doi : 10. 1021/acsami . $7 \mathrm{~b} 09181$.

[21] R. Kumar, P. Lu, X. Xiao, Z. Huang, B. W. Sheldon, Strain-Induced Lithium Losses in the Solid Electrolyte Interphase on Silicon Electrodes, ACS Applied Materials and Interfaces 9 (34) (2017) 28406-28417. doi : 10.1021/acsami.7b06647.

[22] A. L. Michan, M. Leskes, C. P. Grey, Voltage Dependent Solid Electrolyte Interphase Formation in Silicon Electrodes: Monitoring the Formation of Organic Decomposition Products, Chemistry of Materials 28 (1) (2016) 385-398. doi:10.1021/acs . chemmater. 5b04408.

[23] M. Tang, K. Miyazaki, T. Abe, J. Newman, Effect of Graphite Orientation 
and Lithium Salt on Electronic Passivation of Highly Oriented Pyrolytic Graphite, Journal of The Electrochemical Society 159 (5) (2012) A634A641. doi:10.1149/2.073205jes.

[24] T. Kranz, S. Kranz, V. Miß, J. Schepp, B. Roling, Interrelation between Redox Molecule Transport and Li + Ion Transport across a Model Solid Electrolyte Interphase Grown on a Glassy Carbon Electrode, Journal of The Electrochemical Society 164 (14) (2017) A3777-A3784. doi:10. 1149/2.1171714jes.

[25] N. Chapman, O. Borodin, T. Yoon, C. C. Nguyen, B. L. Lucht, Spectroscopic and Density Functional Theory Characterization of Common Lithium Salt Solvates in Carbonate Electrolytes for Lithium Batteries, Journal of Physical Chemistry C 121 (4) (2017) 2135-2148. doi: 10.1021/acs.jpcc. 6 b12234.

[26] J. Maibach, F. Lindgren, H. Eriksson, K. Edström, M. Hahlin, Electric Potential Gradient at the Buried Interface between Lithium-Ion Battery Electrodes and the SEI Observed Using Photoelectron Spectroscopy, Journal of Physical Chemistry Letters 7 (10) (2016) 1775-1780. doi : 10.1021/acs.jpclett.6b00391.

[27] P. Lu, C. Li, E. Schneider, S. Harris, Chemistry, Impedance, and Morphology Evolution in Solid Electrolyte Interphase Films during Formation in Lithium Ion Batteries, The Journal of Physical Chemistry C 118 (2014) 896-903. doi:abs/10.1021/jp4111019.

[28] M. Tang, S. Lu, J. Newman, Experimental and Theoretical Investigation of Solid-Electrolyte-Interphase Formation Mechanisms on Glassy Carbon, Journal of the Electrochemical Society 159 (11) (2012) A1775A1785. doi: 10.1149/2.025211jes.

[29] D. Li, D. Danilov, Z. Zhang, H. Chen, Y. Yang, P. H. L. Notten, Modeling the SEI-Formation on Graphite Electrodes in LiFePO4 Batteries, Journal of the Electrochemical Society 162 (6) (2015) A858-A869. doi:10. $1149 / 2.0161506 j$ jes.

[30] H. J. Ploehn, P. Ramadass, R. E. White, Solvent Diffusion Model for Aging of Lithium-Ion Battery Cells, Journal of The Electrochemical Society 151 (3) (2004) A456. doi : 10.1149/1.1644601.

[31] M. Tang, J. Newman, Electrochemical Characterization of SEI-Type Passivating Films Using Redox Shuttles, Journal of The Electrochemical Society 158 (5) (2011) A530-A536. doi : 10.1149/1.3567765.

[32] M. Tang, J. Newman, Transient Characterization of Solid-ElectrolyteInterphase Using Ferrocene, Journal of the Electrochemical Society 159 (3) (2012) A281-A289. doi:10.1149/2.073203jes.

[33] F. Röder, R. D. Braatz, U. Krewer, Multi-Scale Modeling of Solid Electrolyte Interface Formation in Lithium-Ion Batteries, 26th European Symposium on Computer Aided Process Engineering 38 (1) (2016) 157-162. doi : 10.1016/B978-0-444-63428-3.50031-X.

[34] F. Single, B. Horstmann, A. Latz, Dynamics and morphology of solid electrolyte interphase (SEI), Physical Chemistry Chemical Physics 18 (2016) 17810-17814. doi:10.1039/C6CP02816K.

[35] F. Single, B. Horstmann, A. Latz, Revealing SEI Morphology: In-Depth Analysis of a Modeling Approach, Journal of The Electrochemical Society 164 (11) (2017) E3132-E3145. doi:10.1149/2.0121711jes.

[36] A. A. Tahmasbi, T. Kadyk, M. H. Eikerling, Statistical Physics-Based Model of Solid Electrolyte Interphase Growth in Lithium Ion Batteries, Journal of The Electrochemical Society 164 (6) (2017) A1307-A1313. doi:10.1149/2.1581706jes.

[37] F. Hao, Z. Liu, P. B. Balbuena, P. P. Mukherjee, Mesoscale Elucidation of Solid Electrolyte Interphase Layer Formation in Li-Ion Battery Anode, The Journal of Physical Chemistry C 121 (47) (2017) 26233-26240. doi : 10.1021/acs. jpcc.7b09465.

[38] F. Single, A. Latz, B. Horstmann, Identifying the Mechanism of Continued Growth of the Solid-Electrolyte Interphase, ChemSusChem 11 (12) (2018) 1950-1955. doi:10.1002/cssc. 201800077.

[39] M. Broussely, S. Herreyre, P. Biensan, P. Kasztejna, K. Nechev, R. Staniewicz, Aging mechanism in Li ion cells and calendar life predictions, Journal of Power Sources 97-98 (2001) 13-21. doi : 10.1016 / S0378-7753(01) 00722-4.

[40] J. Christensen, J. Newman, A Mathematical Model for the Lithium-Ion Negative Electrode Solid Electrolyte Interphase, Journal of The Electrochemical Society 151 (11) (2004) A1977. doi:10.1149/1.1804812.

[41] A. M. Colclasure, K. A. Smith, R. J. Kee, Modeling detailed chemistry and transport for solid-electrolyte-interface (SEI) films in Li-ion batteries, Electrochimica Acta 58 (1) (2011) 33-43. doi:10.1016/j . electacta. 2011.08 .067$.
[42] F. Röder, R. D. Braatz, U. Krewer, Multi-Scale Simulation of Heterogeneous Surface Film Growth Mechanisms in Lithium-Ion Batteries, Journal of The Electrochemical Society 164 (11) (2017) E3335-E3344. doi: 10.1149/2.0241711jes.

[43] S. Das, P. M. Attia, W. C. Chueh, M. Z. Bazant, Electrochemical kinetics of SEI growth in Li-ion batteries Part II: Modeling, private communication.

[44] S. Shi, P. Lu, Z. Liu, Y. Qi, L. G. Hector, H. Li, S. J. Harris, Direct calculation of Li-ion transport in the solid electrolyte interphase., Journal of the American Chemical Society 134 (37) (2012) 15476-15487. doi : 10.1021/ja305366r.

[45] F. A. Soto, Y. Ma, J. M. Martinez De La Hoz, J. M. Seminario, P. B. Balbuena, Formation and Growth Mechanisms of Solid-Electrolyte Interphase Layers in Rechargeable Batteries, Chemistry of Materials 27 (23) (2015) 7990-8000. doi:10.1021/acs . chemmater.5b03358.

[46] F. A. Soto, J. M. Martinez de la Hoz, J. M. Seminario, P. B. Balbuena, Modeling solid-electrolyte interfacial phenomena in silicon anodes, Current Opinion in Chemical Engineering 13 (2016) 179-185. doi:10.1016/j.coche.2016.08.017.

[47] Y. Li, K. Leung, Y. Qi, Computational Exploration of the LiElectrode-Electrolyte Interface in the Presence of a Nanometer Thick Solid-Electrolyte Interphase Layer, Accounts of Chemical Research 49 (10) (2016) 2363-2370. doi:10.1021/acs . accounts.6b00363.

[48] O. Borodin, X. Ren, J. Vatamanu, A. Von Wald Cresce, J. Knap, K. Xu, Modeling Insight into Battery Electrolyte Electrochemical Stability and Interfacial Structure, Accounts of Chemical Research 50 (12) (2017) 2886-2894. doi:10.1021/acs .accounts.7b00486.

[49] A. Wang, S. Kadam, H. Li, S. Shi, Y. Qi, Review on modeling of the anode solid electrolyte interphase (SEI) for lithium-ion batteries, npj Computational Materials 4 (1) (2018) 15. doi:10.1038/s41524-018-0064-0.

[50] O. Borodin, M. Olguin, C. E. Spear, K. W. Leiter, J. Knap, Towards high throughput screening of electrochemical stability of battery electrolytes, Nanotechnology 26 (35) (2015) 354003. doi:10.1088/0957-4484/ $26 / 35 / 354003$.

[51] J. M. Martinez De La Hoz, F. A. Soto, P. B. Balbuena, Effect of the electrolyte composition on SEI reactions at $\mathrm{Si}$ anodes of $\mathrm{Li}$ Ion batteries, Journal of Physical Chemistry C 119 (13) (2015) 7060-7068. doi:10.1021/acs.jpcc.5b01228.

[52] K. Leung, Predicting the voltage dependence of interfacial electrochemical processes at lithium-intercalated graphite edge planes, Physical Chemistry Chemical Physics 17 (3) (2015) 1637-1643. doi:10.1039/ c4cp04494k.

[53] O. Borodin, D. Bedrov, Interfacial Structure and Dynamics of the Lithium Alkyl Dicarbonate SEI Components in Contact with the Lithium Battery Electrolyte, The Journal of Physical Chemistry C 118 (2014) 1836218371. doi:10.1021/jp504598n.

[54] O. Borodin, M. Olguin, P. Ganesh, P. Kent, J. Allen, Competitive Lithium Solvation of Linear and Cyclic Carbonates from Quantum Chemistry, Physical Chemistry Chemical Physics 18 (2016) 164-175. doi:10. 1039/x0xx00000x.

[55] S. Bertolini, P. B. Balbuena, Buildup of the Solid Electrolyte Interphase on Lithium-Metal Anodes: Reactive Molecular Dynamics Study, The Journal of Physical Chemistry C 122 (2018) 10783-10791. doi: 10.1021/acs.jpcc.8b03046.

[56] N. Takenaka, Y. Suzuki, H. Sakai, M. Nagaoka, On electrolyte-dependent formation of solid electrolyte interphase film in lithium-ion batteries: Strong sensitivity to small structural difference of electrolyte molecules, Journal of Physical Chemistry C 118 (20) (2014) 10874-10882. doi : 10.1021/jp5018696.

[57] Y. X. Lin, Z. Liu, K. Leung, L. Q. Chen, P. Lu, Y. Qi, Connecting the irreversible capacity loss in Li-ion batteries with the electronic insulating properties of solid electrolyte interphase (SEI) components, Journal of Power Sources 309 (2016) 221-230. doi:10.1016/j.jpowsour. 2016.01 .078$.

[58] M. Nie, D. P. Abraham, Y. Chen, A. Bose, B. L. Lucht, Silicon solid electrolyte interphase (SEI) of lithium ion battery characterized by microscopy and spectroscopy, The Journal of Physical Chemistry C 117 (26) (2013) 13403-13412. doi:10.1021/jp404155y.

[59] K. Leung, F. Soto, K. Hankins, P. B. Balbuena, K. L. Harrison, Stability of Solid Electrolyte Interphase Components on Lithium Metal and Reactive Anode Material Surfaces, Journal of Physical Chemistry C 120 (12) 
(2016) 6302-6313. doi:10.1021/acs .jpcc.5b11719.

[60] K. Ushirogata, K. Sodeyama, Z. Futera, Y. Tateyama, Y. Okuno, NearShore Aggregation Mechanism of Electrolyte Decomposition Products to Explain Solid Electrolyte Interphase Formation, Journal of The Electrochemical Society 162 (14) (2015) A2670-A2678. doi:10.1149/2. 0301514 jes.

[61] S. Shi, Y. Qi, H. Li, L. G. Hector, Defect Thermodynamics and Diffusion Mechanisms in Li2 CO3 and Implications for the Solid Electrolyte Interphase in Li-Ion Batteries, The Journal of Physical Chemistry C 117 (17) (2013) 8579-8593. doi:10.1021/jp310591u.

[62] F. A. Soto, P. Yan, M. H. Engelhard, A. Marzouk, C. Wang, G. Xu, Z. Chen, K. Amine, J. Liu, V. L. Sprenkle, F. El-Mellouhi, P. B. Balbuena, X. Li, Tuning the Solid Electrolyte Interphase for Selective Liand Na-Ion Storage in Hard Carbon, Advanced Materials 29 (18) (2017) 1606860. doi:10.1002/adma. 201606860.

[63] L. Benitez, J. M. Seminario, Ion Diffusivity through the Solid Electrolyte Interphase in Lithium-Ion Batteries, Journal of The Electrochemical Society 164 (11) (2017) E3159-E3170. doi:10.1149/2.0181711jes.

[64] Q. Zhang, J. Pan, P. Lu, Z. Liu, M. W. Verbrugge, B. W. Sheldon, Y. T. Cheng, Y. Qi, X. Xiao, Synergetic Effects of Inorganic Components in Solid Electrolyte Interphase on High Cycle Efficiency of Lithium Ion Batteries, Nano Letters 16 (3) (2016) 2011-2016. doi:10.1021/acs . nanolett. 5 b05283.

[65] D. Bedrov, O. Borodin, J. B. Hooper, Li+ Transport and Mechanical Properties of Model Solid Electrolyte Interphases (SEI): Insight from Atomistic Molecular Dynamics Simulations, The Journal of Physical Chemistry C 121 (30) (2017) 16098-16109. doi : 10.1021/acs.jpcc. $7 \mathrm{~b} 04247$.

[66] M. Tanaka, J. B. Hooper, D. Bedrov, Role of Plasticity in Mechanical Failure of Solid Electrolyte Interphases on Nanostructured Silicon Electrode: Insight from Continuum Level Modeling, ACS Applied Energy Materials 1 (5) (2018) 1858-1863. doi:10.1021/acsaem.8b00344. 